\title{
Über die Vererbung der Haarfarbe der Pferde.
}

\author{
Von 0̈. Winge, Carlsberg Laboratorium, Kopenhagen.
}

(Eingegangen am 26. November 1919.)

\section{Inhaltsverzeichnis.}

1. Einleitung . . . . . . . . . . . . . . . . . . . . . . . 1

2. Die Haarfarben der jütländischen Pferderasse . . . . . . . . . . . . . . 3

3. Bisherige Anschaungen . . . . . . . . . . . . . . . . . . . 5

4. Eigene Untersuchangen . . . . . . . . . . . . . . . . . . . . 9

a) Fuchsfarbige, branne and schwarze Pferde . . . . . . . . . . . . . . 9

b) Geschimmelte und gescheckte Pferde . . . . . . . . . . . . . . . 22

5. Besprechung der Ergebnisse . . . . . . . . . . . . . . . . . 25

6. Znsammenfassung . . . . . . . . . . . . . . . . . . . . . . 31

Literatur . . . . . . . . . . . . . . . . . . . . . . 32

\section{Einleitung.}

Obwohl schon zahlreiche Untersuchungen über die Farbenvererbung bei Pferden vorgenommen worden sind und obgleich viel darüber veröffentlicht worden ist, ist es bisher noch nicht gelungen die Verhältnisse ganz klar zu legen.

Schon aus den achtziger Jahren des vorigen Jahrhunderts liegen sorgfältige Arbeiten vor, namentlich von Crampe, und seither haben in dem jetzigen Jahrhundert eine große Zahl von Forschern, so Pearson, Hurst, Kiesel, James Wilson, Max Müller, Sturtevant, Robertson, Bunsow, Thomann, Ad. R. Walther und Wentworth sich mit dieser Frage beschäftigt und haben mehr oder weniger wertvolle Beiträge zu ihrer Lösung gegeben. Arnold Lang beschäftigt sich in seinem Handbuch „Die experimentelle Vererbungslehre in der Zoologie seit $1900^{\alpha}$ (Jena 1914) so eingehend mit diesen Arbeiten, 
Winge.

indem er die Ergebnisse jedes einzelnen Forschers berichtet, daß ich es unnötig finde, sie hier noch einmal besonders zu besprechen. In dem Folgenden werde ich mich darauf beschränken, die bisher vorliegenden Hauptergebnisse zu nennen, und im übrigen will ich Gewicht auf die Ergebnisse legen, zu denen meine eigenen Studien mich geführt haben, und die zeigen, daß die Sachlage weniger verwickelt ist, als man bisher zu glauben geneigt war - ja sogar so einfach, daß für die Erblichkeitsverhältnisse der wichtigsten Pferdefarben die allereinfachsten Erblichkeitsregeln gelten.

Ebenso wie alle früheren Forscher habe ich auf das "Experiment" insofern verzichten müssen, als ich nur die schon fertig vorliegenden in den Stammbüchern niedergeschriebenen Ergebnisse Anderer benutzen konnte. Hat das Experiment gegenüber dem schon fertig gebucht vorliegenden Stammbüchmaterial den großen Vorteil, daß es dem Untersucher freie Hand läßt bei der Wxhl der gewünschten Paarungen, und daß es ihm die Möglichkeit gibt, eine uneingeschränkte Garantie für die Richtigkeit seiner Ergebnisse zu übernehmen, so haben demgegenüber die Stammbücher den Vorteil, $\mathrm{da} ß$ sie ein ungeheuer großes Material enthalten, wie man es selbst sich nicht würde schaffen können, und $\mathrm{da} ß$ das Material beinahe fertig zur Bearbeitung vorliegt. Im übrigen hängt freilich der Wert des Stammbuchmaterials ganz ab von der Genauigkeit; mit der die Stammbücher geführt sind.

Als ich diese Untersuchungen über die jütländische Pferderasse begann, hegte ich einige Zweifel daran, ob die Stammbücher, die man hier zu Lande geführt hatte, genügend genau wären, um sie mit Erfolg für wissenschaftliche Zwecke benutzen $\mathrm{zu}$ können. - Zu meiner freudigen Überraschung war dieser Zweifel unbegründet und später, in dem Maße wie die Arbeit fortschritt, wurde es mir klar, daß der Verfasser dieser Stammbücher, Staatskonsulent J. Jensen, in ganz erstannlichem Grade Fehler, Verwechselungen und andere Unregelmäßigkeiten vermieden hatte, welche sich bei einer solchen kompilatorischen Arbeit sehr leicht einschleichen. Ich danke auch an dieser Stelle Herrn Staatskonsulent Jensen für die Mitteilungen, die er mir über die Stammbücher und über die Pferdefarben gemacht hat. Auch Fräulein Jeanne B. Möller, Herr stud. mag. M. Hansen und stud. mag. Bengt Dahl, die mich bei den statistischen Arbeiten unterstützt haben, sowie Herrn Premierleutnant F. Lange, der mir bei der Prüfung der Richtigkeit meiner Theorie behilflich war, spreche ich hiermit meinen besten Dank aus. Endlich ist es mir eine angenehme Pflicht aus- 
zusprechen, daß es mir nur dank der Unterstützung durch den Carisberg Fond möglich gewesen ist, meine Untersuchungen durchzuführen.

\section{Die Haarfarben der jütländischen Pférderasse.}

In der jütländischen Pferderasse, der am meisten verbreiteten schweren Rasse in Dänemark, trifft man im wesentlichen die gleichen Farben, welche auch in den Nachbarländern kennzeichnend sind. In der Hauptsache kommen fuchsfarbene, braune und schwarze Pferde, eine Anzahl Schimmel, einige wenige Schecken, und ganz vereinzelte is abellfarbene und weiße Pferde vor. Was uns am meisten interessiert, sind die Erblichkeitsverhältnisse der drei erstgenannten Hauptfarben, dagegen wollen wir absehen von Einzelheiten, wie den weißen Abzeichen, oder den feineren Abstufungen innerhalb der drei Hauptfarben. Daß dies möglich ist, wird sich mit dem Folgenden ergeben.

Das fuchsfarbige Pferd (Chestnut) ist gelbbraun oder rotbraun bis kastanienbraun, selten dunkler. Mähne und Schwanz können von heller und dunkler Färbung sein, sind aber niemals schwarz.

Das braune Pferd kann heller braun (hellbraun, bay) oder dunkler braun (dunkelbraun, brown) bis schwarzbraun sein. Rotbraune Individuen, deren Gesamtfärbung an die fuchsfarbigen erinnert, unterscheiden sich von diesen dadurch, daß Schwanz, Mähne und Ohrenspitzen sowie die Vorderbeine bis zum Knie und die Hinterbeine bis über die Ferse schwarz sind.

Das schwarze Pferd (Rappe) ist ganz schwarz gefärbt. Ganz dunkelbraune (schwarzbraune) Pferde unterscheiden sich von den schwarzen dadurch, daß bei ihnen mindestens die Nasenflügel braun sind.

Werden die Haare der verschieden gefärbten Pferde unter dem Mikroskop untersucht, so soll man nach Wentworth (1914) folgendes beobachten können (S. 10):

"Under both low and high power red pigment granules may be discerned in the sorrel, chestnut, bay or red roan hairs. The granules are sharply distinct and typical in form but there seems also to be a diffuse red, slightly ligther in tinge, distributed quite evenly throughout the cortical layer. This is entirely separate from the effects of spherical aberration, and is quite evidently a basal ground-pigment found in all but white or albino hairs. - Black pigment granules rather larger, coarser and more frequently clustered appear in the black horse. The are so numerous and typical that they quite obscure the red gronnd pigment." 
So einfach wie Wentworth die Sache darstellt, ist sie aber kaum, wenigstens nicht bei der jütländischen Rasse. Eine mikroskopische Untersuchung zeigt zwar deutlich das Vorhandensein von Pigmentkörnern in den Haaren, aber es ist wenigstens nach meiner Erfahrung unmöglich, durch eine Untersuchung des Haarpigments zu unterscheiden ob es sich um ein Haar von einem fuchsfarbenen, braunen oder schwarzen Pferd handelt. Die Sachlage ist folgende:

Die Rinde des fuchsfarbigen Haares zeigt gewöhnlich eine gelbliche Grundfarbe die darauf beruht, daß ein gelbroter Farbstoff in gleichmäßiger Verteilung vorhanden ist. Auf dem gelblichen Grund beobachtet man eine große Menge feiner gelbbrauner etwa $1 \mu$ große Granula, welche oft in Reihe angeordnet sind, oder in länglichen Gruppen zusammenliegen.

Das braune Haar hat eine hellere beinahe ungefärbte Grundsubstanz, in welcher die bräunlichen Pigmentkörner scharf hervortreten. Ihre Verteilung scheint etwas mehr gleichmäßig, ihre Anordnung in Gruppen ist kaum so ausgesprochen, wie bei den Pigmentkörnern des roten Haares, jedenfalls finden sich viele Körner gleichmäßig ausgebreitet.

Das schwarze Haar zeigt dunkelbraune Pigmentkörner auf einem hellen Grund. Die Körner sitzen hier zum Teil sehr einheitlich verteilt und kontrastieren scharf gegen den beinah farblosen Grund.

Kurz gesagt, je dunkler die Haare sind, desto schärfer treten die Pigmentkörner hervor und desto gleichmäßiger scheinen sie verteilt. Je heller die Haare sind, desto weniger kontrastieren die Pigmentkörner gegen den Grund.

Ich habe im Vorstehenden ausdrücklich die Färbungen der Einzelhaare fuchsfarbig, braun und schwarz besprochen und nicht die Gesamthaarfärbungen bei fuchsfarbenen, braunen oder schwarzen Pferden. Bei dem einzelnen Pferd variiert nämlich die Haarfarbe ganz bedentend. Ein fuchsfarbiges Pferd hat oft eine recht große Anzahl dunkler Haare im Schwanz, und diese Haare können bei unmittelbarer Betrachtung und ebenso unter dem Mikroskop ganz das gleiche Aussehen haben wie die Schwanzhaare bei einem schwarzen oder braunen Pferd. Und die Haarfarbe eines fuchsfarbenen Pferdes kann auf der Kruppe oft dunkler und mehr bräunlich sein, als die eines braunen Pferdes. Es ist mir wiederholt begegnet, daß die Leute das bezweifelt haben, aber man überzengt sich leicht davon, indem man Haarproben von fuchsfarbenen sowie von braunen Pferden einsammelt und sie dann nebeneinander legt. Ein braunes Pferd kann auf der Kruppe Haare haben, die viel aus- 
gesprochener fuchsfarbig sind als die eines damit verglichenen fuchsfarbenen Pferdes. Wie schon gesagt liegt der Unterschied zwischen dem braunen und fuchsfarbenen Pferd nicht unmittelbar in der Gesamtfärbung sondern in der Farbe der Mähne, des Schwanzes und der Beine, die bei den erstgenannten schwarz ist, wenn auch oft mit einem rötlichen Schimmer. Dieser glèiche rötliche Schimmer kann gelegentlich auch beobachtet werden im Schwanzhaar schwarzer Pferde.

Wie der Widerspruch zwischen den Beobachtungen Wentworths und mir über das Aussehen des Pigments aufgeklärt werden kann, weiß ich nicht.

Nach meinen eigenen Untersuchungen $m u B$ ich vermuten, daB außer einem gelben Grundpigment, das bei schwarzem und braunem Haar nur schwach, deutlicher aber in fuchsfarbenem Haar sichtbar ist, nur eine Art von Pigment vorhanden ist, welches bei hellerem oder dunklerem Haar einen verhältnismäBig helleren oder dunkleren Ton annimmt. Ein besonders schwarzes Pigment habe ich nicht finden können.

\section{Bisherige Anschaungen.}

Schon in den achtziger Jahren hatte Crampe (nach Lang l. c. S. 747) herausgefunden, da $\mathrm{B}$ :

1. Füchse zeugen miteinander Fuchsfüllen, darunter stichelhaarige Füchse und Fuchsschimmel.

2. Rappen zeugen sowohl Rappen- als Fuchsfüllen, ebenso wie stichelhaarige Füllen und Schimmel mit diesen Farben.

3. Braune Pferde miteinander gepaart bekommen Fällen von allen Farben.

Die Regel, daß Fuchs gepaart mit Fuchs stets fuchsfarbige, niemals braune oder schwarze Fohlen gibt, und daß schwarz gepaart mit schwarz immer nur schwarze oder rote, niemals aber braune Fohlen gibt, hat sich später sehr oft bestätigt, wenn auch die Stammbücher, wobl sicher auf Grund von falschen Eintragungen, seltene Ausnahmen hievon zu zeigen scheinen.

Von den verschiedenen neueren Biologen, die sich mit den Pferdefarben beschäftigt haben, um die Mendelsche Spaltungsregel herauszufinden, von der man glaubte; daß sie für die Vererbung der Pferdefarbe gelten mußte, hat Sturtevant (1912) die Gegenwart folgender Faktoren angenommen. 
1. C Faktor für fuchsrot, der hypostatisch gegenüber allen Farbenfaktoren ist, und der homozygotisch bei allen Tieren vorhanden ist, die nicht Albinos sind.

2. H Faktor für schwarz.

3. B Faktor für braun.

4. G Faktor für Grauschimmel. Grauschimmel werden im Alter weißlich.

5. R Faktor für Dauerschimmel.

Ohne daß wir auf Einzelheiten einzugehen brauchen, muß die Theorie verlassen werden, schon aus dem Grund, daß ein CH-Individuum, da $\mathbf{C}$ als hypostatisch gegenüber $\mathbf{H}$ angegeben wird, schwarz sein müßte. Nichts destoweniger muß Sturtevant, da fuchsfarbig $\times$ schwarz oft braun gibt, annehmen, daB CH-Individuen braun sind.

James Wils on (1910) hatte in Übereinstimmung hiermit und dementsprechend ebenso falsch geglaubt, feststellen zu können, daß braun sei epistatisch gegenüber schwarz, und schwarz gegenüber fuchsfarben (Chestnut).

Ad. R. Walther $(1911,1912)$ glaubte folgende Faktorenpaare zu finden:

Die mit großem Buchstaben bezeichnten Faktoren dominieren.
1. Paar
A. Gelbes Grundpigment,
a. Rotes Grundpigment,

(Grundpigment)

Isabelle oder Hermelin, Fuchs, Alezan, Chestnut Isabelle aux crins blanes, Creamcolour

2. Paar (Epistatisch zu 1)

3. Paar

4. Paar

(Epistatisch zu

1 und 2)
B. Schwarzes Pigment, macht aus Isabelle Falb, (Isabelle aux crins noirs, Dun) und aus Fuchsrot Braun (Bai, Bay and Brown)

C. Braunzeichnung (dominant? unvollständig dominant?). Das schwarze Pigment tritt den Körper nur teilweise bedeckend auf

D. Schimmelzeichnung, auf pigmentierter Haụt stehen zwischen den pigmentierten Haaren untermischt weiße Haare b. Fehlen des schwarzen Pligmentes

c. Rappzeichnung, das schwarze Pigment tritt den Körper völlig bedeckend auf

d. Fehlen der Schimmelzeichnung, d. h. der weißen Haare 
5. Paar E. Scheckzeichnung, e. Fehlen der Scheck-

(Epistatisch zu stellenweise auf unpigmen-

1;2 und 3) tierter Haut unpigmentierte

Haare

6. Paar $\quad$ F. Schabrackenscheckung.

(Epistatisch zu

1, 2 und 3)

Wie daraus hervorgeht, hat Walther keinen Faktor für braune Farbe, indem er braun für eine Vermischung von schwarz und rot annimmt. Auf der anderen Seite hat er seine Zuflucht zur Annahme eines Lokalisationsfaktors $\mathbf{C}$ genommen, entsprechend dem von Castle für die Nager aufgestellten, dessen Vorhandensein schwarze Pferde zu braunen macht, indem die schwarze Farbe eingeschränkt wird auf Schwanz, Mähne und Beine. - Walthers Theorie ist sinnreich aufgebaut, aber bei ihrer Benutzung stößt man doch auf Widersprüchè. Schwarz $\times$ fuchsfarben gibt in der Praxis gewöhnlich eine große Menge brauner Nachkommen. Da braune Individuen nach Walther immer den Lokalisationsfaktor $\mathbf{C}$ enthalten, müssen a priori entweder die schwarzen oder die roten Eltern sehr häufig diesen Faktor wenigstens heterozygotisch enthalten. Die schwarzen können das jedoch nicht sein, da falls im übrigen schwarze Tiere den Faktor $\mathbf{C}$ enthalten, sie dadurch braun werden. Also bleibt nach Walthers Theorie nur die Möglichkeit übrig, daß. die fuchsfarbigen Pferde sehr oft, ja sogar der Regel nach, diesen bei ihnen unsichtbaren Faktor $\mathbf{C}$ enthalten. - Aus Walthers Arbeit geht nicht hervor, worauf übrigens schon Lang aufmerksam gemacht hat, wie Individuen von der Formel aa BB ce oder aa Bb cc aussehen. Ich bin jedoch nicht im Zweifel, daB Walther diese Tiere als schwarz angeben will, obwohl wie er übrigens an einer anderen Stelle selbst ausspricht, schwarze Individuen mindestens einmal $\mathbf{A}$ und einmal $\mathbf{B}$ und stets cc enthalten. Diese Annahme ist ebenfalls die einzige, die mit der praktischen Erfahrung stimmen kann, denn schwarze Pferde enthalten überhaupt selten den Isabellefaktor $\mathbf{A}$.

Walthers Theorie ist insofern von großem Interesse, als sie ein typisches Beispiel einer verschnörkelten Theorie darstellt, die, so unwahrscheinlich sie sich anhört, doch nicht ganz widerlegt werden kann. Wie ich gleich zeigen werde, ist es im wesentlichen Walthers Erklärung der Wirkungsweise seiner Faktoren, die unannehmbar ist:

In neweren Veröffentlichnngen aus dem Gebiet der Vererbungsforschung sieht man mendelnde Faktoren aufgestellt, welche höchst 
eigentümlich wirken, in dem sie bald andere Faktoren hindern, bald hemmen, bald verstärken. Es ist meine Überzengung, daß in vielen Fällen, wenn auch gewiß nicht in allen, es möglich wäre, die aufgestellten Theorien dadurch zu verschönern, daß man sie weniger verwickelt macht. Schon die Bezeichnungsweise der Wirkung der Faktoren ist eine ganz wichtige Sache. Es ist nicht gleich leicht fablich, wenn z. B. das Eigenschaftspaar: glatt-behaart dadurch bezeichnet wird, daB ein gegebener Faktor $\mathbf{A}$ "nackte Epidermis bewirkt" oder aber daß er „Behaarung auf der Epidermis verhindert“. Es kommt nicht bloB dårauf an, daß die Formulierung logisch ist. Es ist ja gewiß ebenso logisch richtig zu sagen: „A verhindert das Fehlen des Mangels an Haaren", aber diese Ausdrucksweise ist unnötig gedankenverwirrend. Daß Walthers Theorie in einem gewissen Grad unter solcher Schwerverständlichkeit leidet, werde ich gleich nachher zeigen. Zunächst soll bloß noch die von Wentworth (1914) besprochen werden. Der Verfasser rechnet mit folgenden Faktoren:

C - der Faktor für das rote oder gelbe Grundpigment.

H - der Faktor für das schwarze Pigment, epistatisch zu C.

B - eip Lokalisationsfaktor; er beschränkt die Bildung des schwarzen Pigmentes auf die Augen, die Mähne, den Schweif und den unteren Teil der Beine.

$\boldsymbol{G}$ - Faktor für gewöhnliches Schimmelhaar (Gray), ist epistatisch zn Fuchsrot, Braun und Dun, nicht zu den Faktoren D und B.

R - Faktor für Roan (Rotschimmelung, Blauschimmelung, d. h. Dauerschimmelung). Dominiert über alle anderen Pigmentfaktoren und ist von der Farbe unabhängig. Rotschimmel sind wahrscheinlich hellbraune und Blauschimmel schwarze Pferde mit dem Roanfaktor $R$.

D - Faktor für Apfelung (Dappling). Besonders beim gewöhnlichen Grauschimmel.

S - Faktor für die Abzeichen (Blesse oder Sterne im Gesicht, weiße Füße).

P - Faktor für Scheckigkeit, Walthers Schabrackenscheckung (Piebald und Skewbald Markings).

M - Faktor für hell cremefarbiges Mähnen- und Schwanzhaar (Light creamy yellow Mane and Tail).

I - Ein dominanter Vedünnungsfaktor; $\mathrm{i}$ - intensive Pigmentierung. I macht schwarz zu mäusefarbig (Mouse), 
Wie daraus hervorgeht, sind die Faktoren von Wentworth im wesentlichen die gleichen wie Walthers, jedoch nimmt er nicht wie dieser an, daß fuchsrote Farbe das Allelomorph für die Isabellafarbe ist. Fuchsfarbig wird als besonderer Faktor $\mathbb{C}$ aufgestellt. Da indessen C immer homozygotisch vorhanden ist, muß es ganz außerhalb der Betrachtung gelassen werden. Wenn dieser Faktor wie bei Walther in das Schema mit hineingenommen wird, muß gleichzeitig Rechenschaft dafür gegeben werden, wie Individuen mit $\mathbf{c H b}$ oder mit $\mathbf{c H B}$ aussehen. ch b sind wohl Albinos, aber die Existenz der zwei zuerst genannten Sorten von Tieren kennt man sicher nicht.

Es ist nicht richtig, wenn Wentworth (und übrigens auch Lang) sagt, daß seine Theorie die erste sei, welche erklären kann, weshalb soviele braune Tiere aus der Kreuzung schwarz $\times$ fuchsfarbig hervorgehen, denn schon Walther, der den Lokalisationsfaktor einführte, konnte damit das Phänomen erklären, indem er annahm, daß die fuchsfarbigen Pferde diesen Lokalisationsfaktor kryptomer enthalten.

Die Arbeit von Wentworth bedeutet wohl Fortschritte gegenüber Walther. Die neuen Faktoren, die er einführt, D für Apfelungen (dappling pattern), S für Abzeichen, d. h. Blesse oder Stern im Gesicht oder weiße Füße, I einen Verdünnungsfaktor usw. sind aber noch so unzulänglich untersucht, daß überhaupt nichts über ihren Wert gesagt werden kann.

\section{Eigene Untersuchungen.}

\section{a) Fuchsfarbige, braune und schwarze Pferde.}

Die Theorie, die ich anstatt der bisher besprochenen für die Vererbungsverhältnisse der fuchsfarbigen, braunen und schwarzen Pferde die selteneren Farben werde ich erst später berühren - aufstelle, ist sehr einfach.

Abgesehen von dem Erbmaterial, das allen Pferden gemeinsam ist, welche keine Albinos sind, ziehe ich nur zwei spaltende Grundunterschiede oder Faktorenpaare in Betracht:

$\mathbf{R}$ ist der Faktor für fuchsrot, $\mathbf{r}$ Fehlen dieses Faktors ${ }^{1}$ ).

$\mathbf{S}$ ist der Faktor für schwarz, s Fehlen dieses Faktors.

Der Klarheit wegen anwende ich hier die "Präsenz-Absenz-Theorie". Natürlich kann man auch $\mathbf{r}$ einen Faktor (Allelomorph $\mathbf{R}$ ) bedeuten lassen. Das spielt ja hier keine Rolle, wenn man nicht mit multiplen Allelomorphen arbeitet. 
Es gibt also folgende vier Sorten von Gameten:

$$
\mathbf{R S}-\mathbf{R s}-\mathbf{r S}-\mathbf{r s}
$$

und die folgenden diploiden Kombinationen können hiervon gebildet werden :

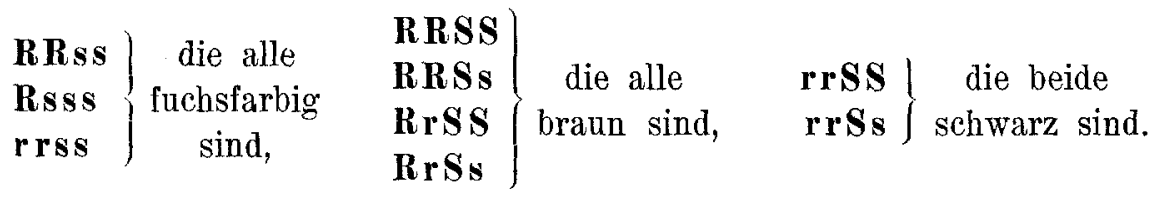

Die Formeln, welche sowohl $\mathbf{R}$ wie $\mathbf{S}$ (einfach oder doppelt) enthalten, stellen braune Tiere dar. Indem eine intermediäre Farbe zwischen fuchsfarbig und schwarz entsteht. Formeln, die nur $\mathbf{S}$ enthalten, bezeichnen schwarze Tiere, und solche, die nur $\mathbf{R}$ enthalten, fuchsfarbige Tiere. Die ganz rezessive Formel rrss läßt die rezessive Grundfarbe sichtbar werden, und die ist ebenfalls fuchsfarbig. Es ist also der Unterschied zwischen der ebenfalls fuchsfarbigen Grundfarbe und der eigentlichen Fuchsfarbe der, daß die erstgenannte gegenüber schwarz ganz rezessiv ist, während die letztgenannte sich mit schwarz zu der Mittelfarbe braun mischt. Wie schon gesagt, sind jedoch braune Pferde nicht einheitlich braun, indem das schwarze Element nur in Schwanz, Mähne und in den Beinen deutlich sichtbar wird.

Es gibt darnach vier Arten braune, 3 Arten fuchsfarbige und zwei Arten schwarze Pferde, die wir in folgendem näher betrachten wollen.

Um zunächst $z u$ kontrollieren, $o b$ die Theorie mit den gewöhnlichen, wohlbekannten Hauptregeln stimmt, so ist leicht zu sehen:

1. Fuchs $\times$ Fuchs gibt nur Fuchsfohlen, indem der Faktor $\mathbf{S}$ bei einer derartigen Paarung niemals hineingebracht wird. Bei der statistischen Durchzählung der Füllen ${ }^{1}$ ), die aus zwei Fuchsalten gefallen sind, zeigt es sich, daß 2480 Paarungen von Fuchs $\times$ Fuchs nach den Stammbüchern 2473 Fuchsfüllen, 7 braune und keine schwarze gegeben haben. Zweifellos sind die 7 braunen auf Falschbenennungen oder auf ungenaue Buchführungen zurückzuführen. Ich bemerke so, daß unter diesen 7 Tieren zwei "kupferbraune" und ein "rotbraunes" Füllen sind, unter welcher Bezeichnung sich sicher richtige Füchse verbergen. Dies ist umsomehr wahrscheinlich, als diese beiden obengenannten Farbenbezeichnungen im ganzen genommen, nur selten in Stammbüchern angewendet werden. Drei davon werden einfach als „braun" bezeichnet und eins als "dunkelbraun", aber dieses letzte, welches, wie gesagt,

1) „Füllen" bedeutet hier und in folgendem nur "Nachkommen", die registrierten Tiere sind alle ausgewachsen. 
Fuchseltern hat, hat nach den Stammbüchern eine Vollschwester, welche hellfuchsfarbig gefärbt ist.

2. Schwarz $\times$ schwarz kann, wie man sieht, nur schwarze und fuchsfarbige Tiere geben. Die Letztgenannten haben dann alle die Formel rrss. Braune Tiere können nicht entstehen, weil der Faktor $\mathbf{R}$, der sich in allen braunen Tieren findet, bei den schwarzen fehlt und so keinesfalls in der Nachkommenschaft von schwarzen Tieren auftreten kann.

Die Zusammenstellung in den Stammbüchern zeigt, daB aus 154 Paarungen aus schwarz $\times$ schwarz 133 schwarze und 21 fuchsfarbige und keine braune Füllen gefallen sind.

3. Braun $X$ braun wird nach der Theorie Nachkommenschaft von allen drei Hauptfarben geben können, weil die Eltern hier sowohl $\mathbf{R}$ wie $\mathbf{S}$ enthalten, zum Teil aber nur den einen oder auch beide Faktoren heterozygotisch.

Die Zählung ergibt, daß aus 2008 braunen Elternpaaren 396 fuchsfarbige, 1480 braune und 132 schwarze Füllen gefallen sind.

Hinsichtlich der allgemeinen Hauptregeln besteht also volle Übereinstimmung zwischen Theorie und Praxis. Aber natürlich muB die Theorie noch eine nähere Prüfung bestehen, ehe sie als gültig angenommen werden kann. Ich setze deshalb im folgenden $z u$ diesem Zweck die Reihe der Beweise fort.

4. Braune Pferde von der Formel RRSS müssen notwendig sowohl einen R- und einen S-Faktor auf alle ihre Nachkommen übertragen, welche deshalb in allen Fällen braun sein müssen. Tiere von dieser Formel müssen, wie wohl leicht eingesehen werden kann, zwei braune Eltern gehabt haben.

Es ist gelungen, in deu Stammbüchern mehrere derartige Hengste nachzuweisen, während Stuten, die ja nur ein wenig zahlreiche Nachkommenschaft ergeben, schwieriger zu analysieren sind.

Der jütländische Hengst 295 Nobel, der mittelbraun und ohne Abzeichen war, hatte diese Formel. Seine beiden Eltern waren braun, nämlich eine mittelbraune Stute und der braune Hengst $160 \mathrm{Gammel}$ Ballehingst. In den Stammbüchern sind 52 Fälle aufgezählt, welche von dem Hengst $295 \mathrm{Nobel}$ abstammen. Sie sind alle braun.

Mit 11 schwarzen Stuten gab er $\begin{cases}5 & \text { braune Füllen. } \\ 6 & \text { dunkelbraune bis schwarze Füllen. }\end{cases}$

mit 11 dunkelbraunen bis $\{6$ braune Füllen, schwarzbraunen Stuten gab er 5 dunkelbraune Fïllen, 
mit 13 braunen Stuten gab er $\left\{\begin{array}{l}1 \text { hellbraunes Füllen, } \\ 12 \text { braune Füllen, }\end{array}\right.$

mit 6 Fuchsstuten gab er

1 hellbraunes Füllen, 4 braune Füllen,

1 dunkelbraunes Füllen,

mit 6 Schimmelstuten gab er $\left\{\begin{array}{l}3 \text { braune Füllen, } \\ 3 \text { dunkelbraune bis schwarzbraune Füllen, }\end{array}\right.$ mit 5 Stuten von unbekannter $\int 3$ braune Füllen,

Farbe gab er $\{2$ dunkelbraune Füllen.

Von anderen jütländischen Hengsten mit dieser Formel kann weiter genannt werden einer von den Söhnen dieses eben besprochenen braunen Hengst $295 \mathrm{Nobel}$, nämlich der Hengst 356 Nobel II, der aus einer dunkelbraunen Stute geboren war. Dieser Hengst hat 26 in den Stammbüchern genannte Füllen, nämlich:
8 aus roten Stuten,
11 aus braunen Stuten,
5 aus schwarzen Stuten,
2 aus Stuten unbekannter Farbe.

Diese 26 Füllen waren alle braun.

Ebenso hatte der dunkelbraune Hengst 398 Bisgaard, der aus zwei braunen Eltern stammt, sicher die Formel RRSS, denn von seinen 24 in den Stammbüchern besprochenen Füllen waren 23 braun und eines ein Schimmel. Da der Schimmelfaktor sowohl über $\mathbf{S}$ wie $\mathbf{R}$ dominiert, ist das Schimmelfüllen, welches aus einer Schimmelstute geboren war, kein Beweis gegen die Richtigkeit der Formel. Von den Müttern dieser ebengenannten 24 Füllen waren 2 Fuchsstuten, 21 braune Stuten und 1 Schimmelstute.

In gleicher Weise können in dem mir vorliegenden Material noch mehrere Hengste bezeichnet werden, die ausschlieBlich branne Füllen gegeben haben, aber die Anzahl der in den Stammbüchern aufgeführten Füllen ist selten groß genug, um daraus mit voller Sicherheit Schlüsse auf die Richtigkeit der Formel ziehen zu können.

5. Als Gegenstïck zu den ebengenannten doppelt homozygotischen braunen Pferden müssen auch Pferde von der Formel rrss, d. h. rezessive Füchse, vorkommen. Es ist ganz klar, daß wenn ein solcher Hengst mit Fuchsstuten gepaart wird, die ja Gameten von den Formeln Rs und rs bilden, stets rote Nachkommen entstehen müssen. Gepaart mit schwarzen Stuten, deren Gameten $\mathbf{r} \mathbf{S}$ oder $\mathbf{r s}$ sind, werden Tiere 
von den Formeln rrSs und rrss d. h. also teils schwarze, teils fuchsfarbige, aber niemals braune Tiere sich ergeben.

Um nun in den Stammbüchern rrss-Hengste zu finden, die ja sowohl aus braunen, als fuchsfarbigen oder schwarzen Eltern entstehen können, oder auch aus. Kreuzungen zwischen Tieren mit diesen Farben, bin ich den sichersten Weg gegangen, indem ich solche Fuchshengste gesucht habe, die aus zwer schwarzen Eltern gefallen sind. Da schwarze Tiere nur Gameten von den Formeln rS oder rs bilden können, ist es nach der Theorie gegeben, daB alle rote Nachkommen aus zwei schwarzen Eltern die Formel rrss haben müssen.

In der jütländischen Rasse gibt es indessen nur verhältnismäßig wenig schwarze Tiere. Im Jahre 1891 war nach Staatskonsulent J. Jensens Aufzählungen das Verhältnis zwischen der Anzahl von Füchsen, Braunen und Rappen ungefähr wie: 26:60:9. Im Jahre 1910 war das Verhältnis bedeutend verändert, nämlich wie $55: 35: 7$; und endlich im Jahre 1918 zeigten die Rappen sich noch seltener, während die Füchse weiter zugenommen hatten, nämlich wie $70: 23: 6$. In Übereinstimmung damit, daß schwarz im Verhältnis zu fuchsfarbig in der jütländischen Rasse selten ist, müssen Tiere, die homozygotisch in $\mathbf{S}$ sind, selten sein, während Tiere, die homozygotisch in $\mathbf{R}$ sind, weniger selten sind. Was die braunen Tiere angeht, so muß sich zeigen, daß Tiere von der Formel RRSs oder RrSs viel häufiger sind als solche mit den Formeln RRSS und RrSS. Das soll später noch näher besprochen werden.

Da schwarze jütländische Pferde, wie gesagt, nachgerade selten geworden sind, müssen Paarungen zwischen zwei schwarzen Stammbuchtieren vorzugsweise in den ältesten Jahrgängen der Stammbücher gesucht werden. Hier findet man dann auch den dunklen Fuchshengst 296 Todbjerg, dessen Eltern eine nicht näher bekannte schwarze Stute und der schwarze Hengst 215 Grantorp waren. Diese Eltern müssen nach der Theorie beide die Formel rrSs gehabt haben.

Von den Nachkommen dieses Hengstes 296 Todbjerg habe ich in den Stammbüchern 34 Füllen aufgezeichnet gefunden, nämlich von folgender Herkunft:

mit 11 Fuchsstuten

mit 16 braunen Stuten

mit zwei schwarzen Stuten
11 Fuchsfüllen

$\left\{\begin{array}{l}7 \text { Fuchs- } \\ 5 \text { braune } \\ 2 \text { schwarzbraune } \\ 2 \text { schwarze }\end{array}\right\}$ Füllen

2 schwarze Füllen 
mit einer gelben Stute

mit einer braunen Stute

mit einer mausfalben Stute
1 braunes Füllen

1 Schimmelfüllen

1 schwarzes Füllen

mit zwei Stuten unbekannter Farbe 2 braune Füllen.

Leider ist das Material nicht besonders groB, aber die Paarungen mit den zwei schwarzen Stuten haben in Übereinstimmung mit der Theorie keine braunen Tiere ergeben. Die Paarungen mit gelben oder mausefalben- Stuten, welche ein braunes und ein schwarzes Füllen ergeben haben, zeigen, da 296 Todbjerg ganz rezessiv ist, daß gelb dominiert über braun und mausefalb über schwarz, falls, worauf andere Untersuchungen hindeuten, wir es auch mit einzelnen Grundunterschieden in Hinsicht auf diese Farben zu tun haben.

6. Hengste von der Formel RRss müssen auch an ihren Nachkommen erkennbar sein, denn diese müssen stets $\mathbf{R}$ enthalten und können daher niemals schwarz sein.

Als ein Beispiel kann hier der in der jütländischen Pferdezucht bekannte dunkelfuchsrote, hellmähnige Hengst $445 \mathrm{Munkedal}$ genannt werden. Mankedals beide Eltern waren Füchse, der Vater war der dunkelfuchsrote $\mathbf{3 8 2}$ Valdemar II.

Von Munkedals 90 in den Stammbüchern genannten Nachkommen waren 64 Füchse, 25 Braune und ein Braunscheck. Außerdem wird angegeben, aber zweifellos fehlerhaft, daß ein Füllen $s c h w a r z^{1}$ ) gewesen sein soll. Im einzelnen gab 445 Munkedal:

mit 45 Fuchsstuten

mit 40 braunen Stuten

mit 3 schwarzen Stuten
45 Fuchsfüllen

f 18 Fuchsfüllen

$\{22$ branne Füllen

$\{1$ Fuchsfüllen

$\{2$ braune Füllen

mit 2 Stuten von unbekannter Farbe $\left\{\begin{array}{l}1 \text { braunes Füllen } \\ 1 \text { braungeschecktes Füllen }{ }^{2} \text { ). }\end{array}\right.$

Auch 445 Munkedals berühmter Enkel 839 Aldrup Munkedal hatte die gleiche Formel RRss. Ich habe eine Statistik über die sehr

1) Staatskonsulent Jènsen sagt gerade mit Hinsicht auf diese Stute, dab "angeblich". ("efter Opgivende") Munkedal ihr Vater gewesen sein soll. Das Mißtrauen, das man zwischen den Zeilen liest, ist zweifellos berechtigt, und ich erlaube mir ganz von diesem Fall abzusehen.

2) Die Mutter, die bloß als ,jütländische Stute" bezeichnet ist, muB sicher ein Braun- oder Schwarzscheck gewesen sein, da die Eigenschaft geseheckt dominant über alle drei Hauptfarben ist. 
zahlreiche Nachkommenschaft dieses Hengstes nicht ausgearbeitet. Im Stammbuch XII, 1913 über Stuten jütländischer Rasse hat Staatskonsulent Jensen eine kurze Übersicht über die Nachkommenschaft, 283 Stück, zusammengestellt und nachgewiesen, daß kein Nachkomme schwarz ist.

7. Schwarze Hengste von der Formel rrSS werden ebenso an ihrer Nachkommenschaft erkennbar sein, und als Beispiel für einen solchen Hengst will ich 499 Valdemar Engebjerg nennen. Da die Formel zweimal $\mathbf{S}$ enthält, müssen beide Eltern diesen Faktor enthalten haben, d. h. keines von den Elterntieren kann fuchsfarbig gewesen sein. Valdemar Engebjergs Vater war in Übereinstimmung hiermit schwarz und seine Mutter mittelbraun.

Infolge der Formel rrSS muß dieser Hengst immer einen S-Faktor auf seine Nachkommenschaft übertragen, welche deshalb niemals fuchsfarbig sein kann. Wenn der Hengst gepaart wird mit einer schwarzen Stute, muß die ganze Nachkommenschaft schwarz sein, indem immer .S, aber niemals. $\mathbf{R}$ auf die Nachkommenschaft übertragen wird. Die Erfahrung stimmt hiermit vollständig. Von den Nachkommen des Hengstes 449 Vardemar Engebjerg habe ich die folgenden in den Stammbüchern genannt gefunden, im ganzen 108 Füllen:

$$
\begin{aligned}
& \text { mit } 15 \text { Fuchsstuten }\left\{\begin{array}{l}
9 \text { braune } \\
6 \text { schwarze }
\end{array}\right\} \text { Füllen } \\
& \text { mit } 78 \text { braunen Stuten }\left\{\begin{array}{l}
54 \text { braune } \\
24 \text { schwarze }
\end{array}\right\} \text { Füllen } \\
& \text { mit } 13 \text { schwarzen Stuten } 13 \text { schwarze Füllen } \\
& \text { mit } \left.2 \text { Schimmelstuten }{ }^{1}\right)\left\{\begin{array}{c}
1 \text { schwarzes } \\
1 \text { Schimmel- }
\end{array}\right\} \text { Füllen }
\end{aligned}
$$

8. Ein Beispiel von einem Pferd mit der Formel Rrss werde ich ebenfalls geben. Solche Hengste, die heterozygotisch in einem oder in beiden Faktoren sind, sind schwierig zu analysieren, weil sie in $F_{1}$ in stärkerem Grade spalten, aber wenn Hengste mit einer zahlreichen Nachkommenschaft $\mathrm{zu}$ der Untersuchung verwendet werden, ist doch ein Erfolg möglich.

Der Hengst 339 Aksel hatte diese Formel und war also fachsfarbig. Seine Eltern waren eine Schimmelstute und ein Fuchshengst 259 Glenstrup. Aksels Nachkommenschaft sind folgende 80 Füllen:

1) In Wirklichkeit zwei Paarungen mit derselben Stute. 


\begin{tabular}{|c|c|c|}
\hline mit 20 Euchsstuten & $\begin{array}{l}19 \text { Fuchs - } \\
1 \text { kupferbraunes }{ }^{1} \text { ) }\end{array}$ & $\begin{array}{c}\text { Füllen } \\
n\end{array}$ \\
\hline & 22 Fuchs- & $"$ \\
\hline mit 49 braunen Stuten & $\begin{array}{l}23 \text { braune } \\
4 \text { schwarze }\end{array}$ & $"$ \\
\hline it 8 achwor & 3 Fuchs- & $"$ \\
\hline o scuwarzen slucen & 3 schwarze & $"$ \\
\hline mit 3 Schimmelstuten & $\begin{array}{l}1 \text { Fuchs- } \\
2 \text { schwarze }\end{array}$ & 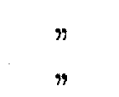 \\
\hline
\end{tabular}

Im Gegensatz zu Fuchshengsten von der rezessiven Formel rrss sieht man, daß dieser Hengst aus schwarzen Stuten anch braune Nachkommen geben kann. Und im Gegensatz zu Fuchshengsten von der Formel RRss hat er auch schwarze Nachkommen.

Wie aus dem vorher Gesagten hervorgeht, spricht alles dafür, daß die aufgestellte Theorie richtig ist. Es ist indessen möglich, sie weiter auf verschiedene Weise zu prüfen. Wie Seite 13 gesagt, ist die schwarze Farbe in der jütländischen Pferderasse stets (wenigstens in dem letzten Menschenalter) die seltenste von den drei Hauptfarben gewesen. Die braune war um das Jahr 1890 die häufigste. In den späteren Jahren hat die Anzahl der Füchse zugenommen auf Kosten der Braunen und der Rappen. Das kann auch folgendermaßen ausgedrückt werden. Der Faktor $\mathbf{S}$ ist stets, wenigstens seit 1890, der seltenere von den beiden gewesen und er ist namentlich in den späteren Jahren seltener geworden. R dagegen ist schon seit dem Jahre 1890 sehr verbreitet, weshalb die Tiere damals am öftesten $\mathbf{R S}$ oder $\mathbf{R} \mathbf{s}$ enthielten. Später als $\mathbf{S}$ durch Selektion unter. den Zuchttieren aus den Formeln mehr und mehr verschwand, bleibt nur $\mathbf{R}$ zurück, was bewirkt, daß die Füchse in die Mehrzahl kommen.

Von den 9 möglichen Formeln

Füchse $\left\{\begin{array}{l}\text { RRss } \\ \text { Rrss } \\ \text { rrss }\end{array} \quad\right.$ Braune $\left\{\begin{array}{l}\text { RRSS } \\ \text { RRSs } \\ \text { RrSS } \\ \text { RrSs }\end{array} \quad\right.$ Rappen $\left\{\begin{array}{l}\text { rrSS } \\ \text { rrSs }\end{array}\right.$

1) Die Mutter wird als "kupferrot" bezeichnet. Die Mutter und das Füllen sind also sicher von derselben Farbe gewesen, sei es daß diese nun fuchsfarbig oder braun gewesen ist. 
sind mit andern Worten die, welche $\mathbf{S}$ zweimal enthalten, verhältnismäßig selten, während die mit zweimal $\mathbf{R}$ nicht selten sind. Es liegt ja in der Natur der Sache, daB eine gewisse Übereinstimmung bestehen muß zwischen der Anzahl Füchsen, Braunen und Rappen und der Anzahl von $\mathbf{R}$ und $\mathbf{S}$ in den Formeln. Bei braunen Tieren werden so RRSS und R rSS recht selten sein im Vergleich za den andern beiden Sorten von braunen RRSs und RrSs. Wir sind imstande, durch die Nachkommenschaft zu prüfen, ob sich das so verhält.

1. Der Fuchshengst $339 \mathrm{Aksel}$ hatte, wie schon besprochen, die Formel Rrss. Er wurde unter anderen mit 49 brannen Stuten gepaart, und ans diesen Paarnngen fielen folgende Füllen:

$$
49 \text { branne Stuten } \times \sigma^{7} \text { Rrss gaben }\left\{\begin{aligned}
22 & \text { Fuchs-Füllen } \\
23 & \text { braune Füllen } \\
4 & \text { Rapp-Füllen. }
\end{aligned}\right.
$$

Welche Formel hatten nun diese 49 Stuten?

Wenn wir eine Wahrscheinlichkeitsberechnung rückwärts vornehmen, kommen wir zu folgendem Ergebnis: 2 RRSS, 3 RrSS, 18 RRS und $26 \mathrm{RrSs}$, was folgendermaßen gezeigt werden kann:

Die Gameten, welche von diesen 49 Staten gebildet werden, ergeben sich aus deni nachstehenden Schema:

\begin{tabular}{r|c|c|c|c} 
Wahrscheinliche Anzahl der Gameten \\
\hline & RS & Rs & rS & rs \\
\hline 2 RRSS & 2 & & & \\
3 R RSS & 1,5 & & 1,5 & \\
18 RRS & 9 & 9 & & \\
26 RrSs & 6,5 & 6,5 & 6,5 & 6,5 \\
\hline
\end{tabular}

Da die Gameten von 339 Aksel zu $50 \%$ Rs und zu $50 \%$ rs sind, kann folgende Aufstellung vorgenommen werden, um das Aussehen der Nachkommenschaft zu beurteilen, man braucht nämlich nur die Multiplikationen auszuführen:

\begin{tabular}{|c|c|c|c|c|}
\hline$\sigma^{7}$ & $19 \mathrm{RS}$ & $15,5 R_{S}$ & $8,0 \mathrm{rS}$ & $6,5 \mathrm{rs}$ \\
\hline $\begin{array}{l}1 / 2 \mathrm{Rs} \\
1 / 2 \mathrm{rs}\end{array}$ & $\begin{array}{c}9,5 \mathrm{RRS} \mathrm{s} \\
9,5 \mathrm{RrSs}\end{array}$ & $\begin{array}{l}7,75 \text { RRss } \\
7,75 \text { Rrss }\end{array}$ & $\begin{array}{c}4,0 \mathrm{RrSs} \\
4,0 \mathrm{rrSs}\end{array}$ & $\begin{array}{l}3,25 \mathrm{Rrss} \\
3,25 \mathrm{rrss}\end{array}$ \\
\hline
\end{tabular}


oder mit anderen Worten $9,5+9,5+4=23$ Braune und $7,75+7,75+3,25+3,25=22$ Füchse sowie $4,0=4$ Rappen

was zeigt, daß die Wahrscheinlichkeitsrechnung richtig ausgeführt ist.

Die Berechnung kann dadurch ausgeführt werden, daß man in dem gegebenen Fall weiß, daß die Anzahl der rS-Gameten, welche von den 49 Stuten gebildet wird, 8 sein muß, damit 4 Rappfüllen aus der Paarung nit 339 Aksel Krss fallen können. In ähnlicher Weise können die übrigen Zahlen berechnet werden.

Falls die unbekannte Zahl von Stuten mit den Formeln

$\begin{array}{rrcc}\operatorname{RRSS} & \mathbf{x} & \text { genannt } & \text { werden } \\ \operatorname{RrSS} & \mathbf{y} & & \\ \operatorname{RRSs} & \mathrm{z} & " & " \\ \text { und } \operatorname{RrSs} & \mathbf{v} & " & ",\end{array}$

kann man leicht sehen, $d a \beta v+y=32$ und $z+v=44$ sein $m u B$, damit 23 Braune, 22 Füchse und 4 Rappen fallen können. Da $z$ und $v$ gerade die Formeln sind, in denen $\mathbf{S}$ heterozygotisch vorkommt, ergibt sich $49 \div(\mathrm{z}+\nabla)=$ Anzahl der Stuten mit SS d. h. $=5$.

Die zwei Gleichungen mit drei Unbekannten können natürlicherweise nicht gelöst werden, aber da RR-Stuten (im Verhältnis zu RrStuten) ja als gleich häufig unter den SS-Tieren wie unter den SsTieren angenommen werden müssen, kann folgendes Verhältnis anfgestellt werden:

$$
\mathrm{x}: \mathrm{y}=\mathrm{z}: \mathrm{v},
$$

womit die gegebene Lösung als die einzig richtige sich ergibt.

49 Stuten von den folgenden Formeln:

1 RRSS, 4 RrSS, 20 RRSs, 24 RrSs,

werden, wie man sieht, gepaart mit 339 Aksel anch 23 braune Füllen, 22 Fuchsfüllen und 4 Rappfüllen ergeben, aber hier ist die Bedingung $\mathrm{x}: \mathrm{y}=\mathrm{z}: \mathrm{v}$ nicht so gut erfüllt, weshalb die erstgenannte Lösung wahrscheinlich die richtigere ist. Auf alle Fälle ändert sich das Hauptergebnis, daß nämlich wahrscheinlich nur 5 von den 49 Stuten SS, dagegen 44 Ss waren, nicht.

Daraus geht mit anderen Warten hervor, daß die Wahrscheinlichkeitsberechnung zu dem Schlusse führt, daß unter den 49 braunen Stuten, mit denen 339 Aksel gepart worden ist, nur 5 SS, dagegen $44 \mathbf{S s}$ waren. SS-Individuen sind sonach verhältnismäßig selten. In dem gegebenen Beispiel bildeten sie nur etwa 10,2\% von allen braunen Stuten. 
2. Zur Kontrolle wollen wir ein anderes Beispiel durch eine solche Berechnung durchprüfen. Seite 13 ist mitgeteilt, daß 296 Todbjerg von der Formel rrss gepaart wurde mit 16 braunen Stuten, woraus 7 braune, 7 fuchsfarbige und 2 schwarze Füllen fielen. Falls alle 4 Formeln von braunen Pferden gleich häufig gewesen wären, würde wahrscheinlich die Nachkommenschaft bestehen aus 9 braunen, 4 fuchsfarbigen und 3 schwarzen Füllen.

Wenn die Anzahl von Stuten von der Formel RRSS $x$ genannt wird, RsSS y, RRSs z, RrSs v, so haben wir $\mathrm{x}+\mathrm{y}=2, \mathrm{z}+\mathrm{v}=14$, $2 y+v=8$, welche Gleichung befriedigt wird von $x=1, y=1, z=8$, $\mathrm{v}=6$. Das Verhältnis $\mathrm{x}: \mathrm{y}=\mathrm{z}: \mathrm{v}$ paßt auch hier sehr gut, wenn man die geringe Zahl Exemplare in Betracht zieht.

Von den 16 braunen Stuten finden wir also nach der Wahrscheinlichkeitsberechnung, daß 2 SS und 14 Ss waren - oder mit anderen Worten $12,5 \%$ von allen Braunen waren homozygotisch in $\mathbf{S}$, wovon man sagen muß, daß es vollauf genügend mit den oben gefundenen $10,2 \%$ stimmt.

Die zwei Hengste stammen aus ungefähr der gleichen Zeit, doch ist 296 Todbjerg, wie die Laufnummer zeigt, etwas älter als $339 \mathrm{Aksel}$, also aus einer Zeit, in der der S-Faktor etwas häufiger war, was mit den Prozentzahlen übereinstimmt. Das zuletzt genannte Material ist von so geringem Umfange, daß es selbstverständlich keine große Beweiskraft hat: man könnte jedenfalls sagen, daß es ein Zufall sei, daß der Prozentsatz etwas höher ist, aber da das Beispiel ganz zufällig gewählt ist, und da es mit dem ersten stimmt, habe ich es mitgenommen.

3. Eine bessere Kontrolle haben wir in der folgenden Berechnung. Der Hengst 445 Munkedal (RRss) wurde gepaart mit 40 braunen Stuten and gab mit diesen 22 brane und 18 fuchsfarbene Füllen.

Die unbekannte Anzahl von braunen Stuten mit der Formel

RRSS sollen $x$ genannt werden

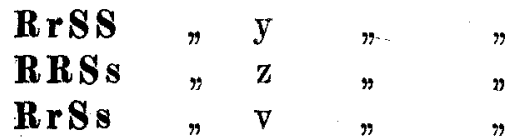

Die Anzahl der verschiedenen Gameten, die diese Tiere bilden, wird dann sein:

$$
\begin{array}{rll}
\text { von } & \mathbf{R S} & \mathrm{x}+1 / 2 \mathrm{y}+1 / 2 \mathrm{z}+1 / 4 \mathrm{v} \\
\Rightarrow & \mathbf{R S} & 1 / 2 \mathrm{z}+1 / 4 \mathrm{v} \\
\Rightarrow & \mathbf{r S} & 1 / 2 \mathrm{y}+1 / 4 \mathrm{v} \\
& \text { rs } & 1 / 4 \mathrm{v} .
\end{array}
$$


Da die Gameten von 445 Munkedal alle Rs sind, werden also die Nummern 1 und 3 von den Gametenformeln der Stuten braune Tiere geben, die Nummern 2 und 4 dagegen fuchsfarbene. Für die letztgenannten haben wir also:

$$
1 / 2 \mathrm{z}+1 / 4 \mathrm{v}+1 / 4 \mathrm{v}=18, \text { oder } \mathrm{v}+\mathrm{z}=36
$$

da $\mathrm{v}+\mathrm{z}$ die Formeln mit $\mathbf{S}$ s sind, wogegen $\mathrm{x}+\mathrm{y}$ die Formeln mit SS sind, müssen von den 40 braunen Stuten, mit denen Munkedal gepaart war, $36 \mathrm{Ss}$, dagegen nur $4 \mathrm{SS}$ in der Formel gehabt haben. $10 \%$ von den braunen Stuten müssen also $\mathbf{S}$ homozygotisch enthalten haben. Wir finden somit zum dritten Male, daß S S-Tiere nur 10-12\% aller braunen Tiere ausmachen.

4. Auch das Verhältnis zwischen der Anzahl der R R- und der $\mathbf{R} \mathbf{r}$-Pferde zu untersuchen ist von Interesse. Wie es sich auf Seite 17 bei der Ausrechnung der Nachkommenschaft von 339 Aksel ergab, kamen wir hier auf einem Weg zu dem Schluß, daß von den braunen Stuten, mit denen dieser Hengst gepaart war, 20 die Formel $\mathbf{R} \mathbf{R}$ und $29 \mathbf{R} \mathbf{r}$ hatten, d. h. zirka 40,8\% waren homozygotisch in Hinsicht auf $\mathbf{R}$. Diese Berechnung war, theoretisch gesehen, nicht sehr genau.

Wir wollen jetzt auf einem anderen Weg dieses Ergebnis prüfen, indem wir das Material von den Paarungen des schon besprochenen Hengstes Valdemar Engebjerg (rrSS) mit 78 braunen Stuten benützen. Diese Stuten gebaren 54 braune und 24 schwarze Füllen. Falls RR gleich häufig gewesen wäre, wie $\mathbf{R} \mathbf{r}$, wären $75 \%$ von den Nachkommen braun und 25\% schwarz gewesen. Wenn $\mathbf{R R}$ ganz gefehlt hätte, wären $50 \%$ braun und $50 \%$ schwarz gewesen. Wenn $\mathbf{R r}$ gefehlt hätte, wären alle Füllen braun gewesen. Wir benützen die obenstehende Aufstellung (S. 19) noch einmal und sehen daraus, daß die beiden untersten Gametenformeln die beiden einzigen sind, die zusammen mit IS schwarze Nachkommen geben werden, daher:

$$
1 / 2 y+1 / 4 v+1 / 4 v=24, \text { d. } h . y+v=48 \text {. }
$$

Wir finden also, daß 48 von den braunen Stuten $\mathbf{R} \mathbf{r}$ und $30 \mathbf{R R}$ enthalten, oder mit anderen Worten rund $38,5 \%$ sind homozygotisch im Hinblick auf den Faktor $\mathbf{R}$, was ja unbestreibar mit dem obengewonnenen Ergebnis übereinstimmt. Es ist nicht möglich, aus diesem Beispiel das Verhältnis zwischen den SS- und den Ss-Tieren zu berechnen; da wir aber früher schon gefunden haben, daß das Häufigkeitsverhältnis dieser Formeln ungefähr gleich $1: 9$ war, sind die 78 braunen Stuten, mit denen 449 Valdemar Engebjerg gepaart war, wahrscheinlich die folgenden gewesen:

3 RRSS, 27 RRSs, 5 RrSS und 43 RrSs. 
Wenn von den braumen Pferden $40 \%$ RR führen und $10 \%$ SS, dann müssen also von 100 braunen Pferden die verschiedenen Formeln mit dieser Häufigkeit auftreten: 4 RRSS : 36 RRSs : 6 RrSS : 54 RrSs. - Wir denken uns jetzt solche Pferde willkürlich untereinander gepaart; die dadurch erzeugten Füllen werden die folgenden sein: $72,5 \%$ braune : $20,2 \%$ fuchsfarbige : $7,2 \%$ schwarze, was sich sehr leicht ausrechnen läßt, - und dies stimmt nun sehr hübsch mit den beobachteten Zablenverhältnissen, siehe die Tabelle S. 30): 1480 (73,7\% braune : 396 $(19,7 \%)$ fuchsfarbige : $132(6,6 \%)$ schwarze.

In ähnlicher Weise wie eben gezeigt, kann man allerhand andere Berechnungen über die Formeln anderer Stammbuchtiere vornehmen, und in keinem Fall, wo ich das ausgeführt habe, sind Inkonsequenzen oder Widersprüche herausgekommen, wie sie unweigerlich hätten kommen müssen, wenn die Theorie falsch wäre.

In Nachstehendem folgt eine Liste über die Farben und Formeln von einer Anzahl der bekanntesten jütländischen Stammbuchhengste (teils die schon genannten; teils andere), deren Formeln ich berechnet habe.

\begin{tabular}{|c|c|c|c|c|c|}
\hline & & & & Farbe & $\begin{array}{l}\text { Formel } \\
\text { re Ss }\end{array}$ \\
\hline 215 & Granthorp & & & schwarz & $\begin{array}{l}\text { rrSs } \\
\text { RRSS }\end{array}$ \\
\hline 295 & Nobel. & . & & mittelbraun & RRSS \\
\hline 296 & Todbjerg & & & ankelfuchs & r rss \\
\hline 339 & Aksel & & & hs & Rrss \\
\hline 398 & Bisg & & & an & RRSS \\
\hline 445 & Mun & & & as & R R s s \\
\hline 449 & Valdemar Engebje & erg & & sch & $\operatorname{rrSS}$ \\
\hline 669 & r Engebje & erg & IV & mitte & RrSs \\
\hline 839 & Munkedal & & & $\mathrm{fu}$ & RRss \\
\hline 000 & f Jylland & & & hellf & Rrss \\
\hline 5 & Hövding & & & hellf & Rrss \\
\hline $15 !$ & Leif & & & $\mathrm{fu}$ & RRs s \\
\hline 1263 & Hövding & & & dunke. & RRSs \\
\hline 126 & Hövding & & & & Rrss \\
\hline 1269 & leur. & & & & Rrss \\
\hline 133 & As & & . & $\operatorname{sch}$ & $\mathbf{r S S}$ \\
\hline 1346 & $\mathrm{Kr}$ & & & dunk & $\mathbf{R r S s}$ \\
\hline 1367 & Valdemar Kran & & & dunkelbraun & RrSs \\
\hline
\end{tabular}

Aus dieser Liste wird sich deutlich ergeben, daß nicht etwa eine bestimmte Formel einer bestimmten Nuance innerhalb der Hauptfarben 
braun oder fuchsfarbig entspricht. Dunkelfüchse können sowohl die Formel RRss, wie auch Rrss und rrss haben, ebenso können sowohl dunkelbraune wie mittelbraune beide die Formel RrSs und RR SS haben. Das bedentet natürlich, daß außer den hier genannten Faktoren $\mathbf{R}$ und $\mathbf{S}$ es noch andere gibt, die für die Nuance bestimmend sind, Intensitätsfaktoren, Verdunkelungsfaktoren oder ähnliches; diese habe ich nicht klarzulegen versucht, weil die Stammbücher so, wie sie bisher geführt worden sind, kaum ins einzelne zu gehen erlauben. Damit das Stammbuchmaterial dafür verwendet werden könnte, müßten die Nuancen innerhalb der Hauptfarben immer angegeben werden, aber dies ist durchaus nicht immer der Fall. Zweifellos sind z. B. nicht alle Pferde, welche als "Füchse" bezeichnet sind, alle von derselben Fürbung. Es wäre von Bedeutung für die Klarlegung der Nuancierungsfaktoren, wenn die Stammbücher eine mehr ins einzelne gehende Farbenbezeichnung immer anwenden würden, und das beste wird es sein, wenn man hier eine Farbentafel bei der Beurteilung anwenden könnte, um so weit als möglich die individuelle Beurteilung zu vermeiden, welche, wie bekannt, innerhalb der feineren Abstufungen recht unzuverlässig ist.

\section{B. Schimmel und Schecken.}

Nur eine ganz geringe Zahl von Pferden der jütländischen Rasse ist weder fuchsartig noch braun, noch schwarz, doch sind in den Stammbüchern eine genügend große Zahl von Schimmeln und Schecken enthalten, um zeigen zu können, daß diese Farben dominant sind, wie dies schon längst bei anderen Pferderassen nachgewiesen worden ist. Da diese Sache als klargelegt angesehen werden mnß, will ich nur durch eine kurze Übersicht und ein paar Beispiele die GesetzmäBigkeit beleuchten:

Weibliche Nachkommen.

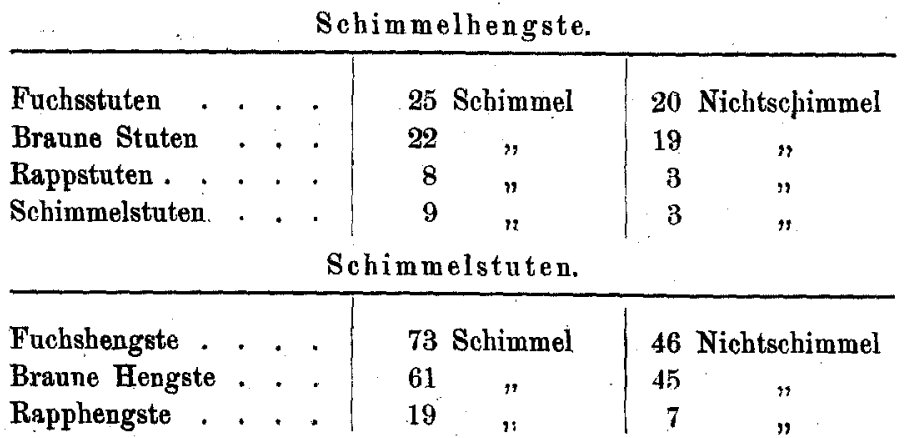


Männnliche Náchkommen.

\begin{tabular}{l|c|c|}
\hline Schimmelhengst $\times$ Schimmelstute .... & 2 Schimmel & 1 Nichtschimmel \\
$\begin{array}{c}\text { Nichtschimmelhengste und Nichtschimmelstuten } \\
\times \text { Schimmelhengste und Schimmelstuten . . }\end{array}$ & $32 \quad$, & $38 \quad "$,
\end{tabular}

Aus der obenstehenden Übersicht ergibt sich, daß aus den Paarungen zwischen Schimmeln und Nichtschimmeln gegen 60\% Schimmel gefallen sind. $\mathrm{Da} \beta$ der Prozentsatz nicht 50 ist, sondern mehr, wird natürlich teils durch den Umstand verursacht, daß eine gewisse Anzahl von Elternindividuen ho mozygotische Schimmel gewesen sind und demnach in ihrer Nachkommenschaft lauter Schimmel gegeben haben, aber zum Teil kann es auch daherrühren, daB die Schimmelfarbe ansprechend gefunden worden ist, so. daß vielleicht aus diesem Grunde unverhältnismäßig viele Schimmel in die Stammbücher aufgenommen worden sind.

$\mathrm{Daß}$ der Schimmelfaktor nicht rezessiv ist, geht anch aus der Erfahrung hervor, daß Schimmel niemals aus zwei Nichtschimmeln ') fallen, wogegegen zwei Schimmel sehr gut Nichtschimmel als Nachkommen haben können, wie teils die obenstehenden Tabellen, teils der folgende kleine Stammbaum zeigt.

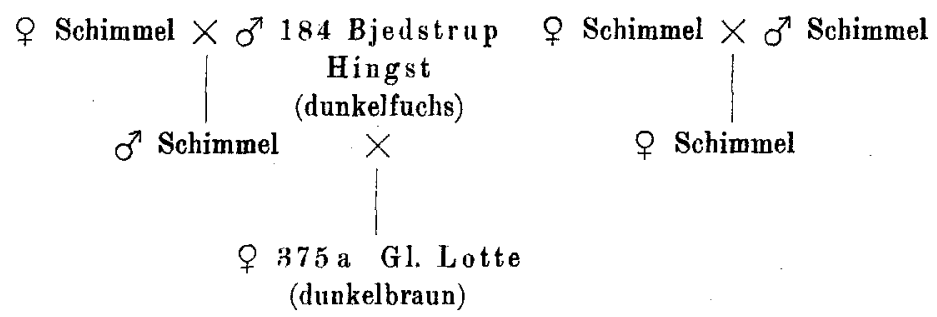

Die beiden Schimmel, deren Nachkommen die dunkelbraune Stute 375 a Gl. Lot te ist, müssen selbstverständlich heterozygotische Schimmel gewesen sein, und das gleiche gilt für die allermeisten Schimmel, welche in die Stammbücher der jütländischen Pferde aufgenommen sind.

$1049 \mathrm{Hads}$ Herreds Munkedal war ebenso ein heterozygotischer Schimmel und sollte dementsprechend bei der Paarung mit Nichtschimmelstuten $50 \%$ Schimmel und $50 \%$ Nichtschimmel als Nachkommenschaft ergeben. In den Stammbuichern habe ich ton ihm Nachkommenschaft aus im ganzen 41 Paarungen mit Nichtschimmelstuten

1) Hier ist nur die Rede von Grauschimmeln. Für die sogenannten Rotschimmel scheint die Sachlage bisweilen anders zu sein, aber das Material ist nicht grob genug, um ein Urteil zu gestatten. 
aufgeführt vorgefunden. Von diesen 41 Nachkommen waren 22 Schimmel und 19 Nichtschimmel.

Eine nähere Analyse von den in den Schimmeln steckenden Faktoren für schwarz und fuchsfarbig kann vorgenommen werden, wenn genügend zahlreiche Nachkommen da sind. Eine Analyse der nichtgeschimmelten Töchter (Söhne sind überhaupt keine da) von 1049 Hads Herreds Munkedal zeigt, daß, wenn nicht der Schimmelfaktor die Wirkung der anderen Faktoren verdeckt hätte, der Hengst braun gewesen wäre. Der Hengst 1049 hat folgende Nachkommen:

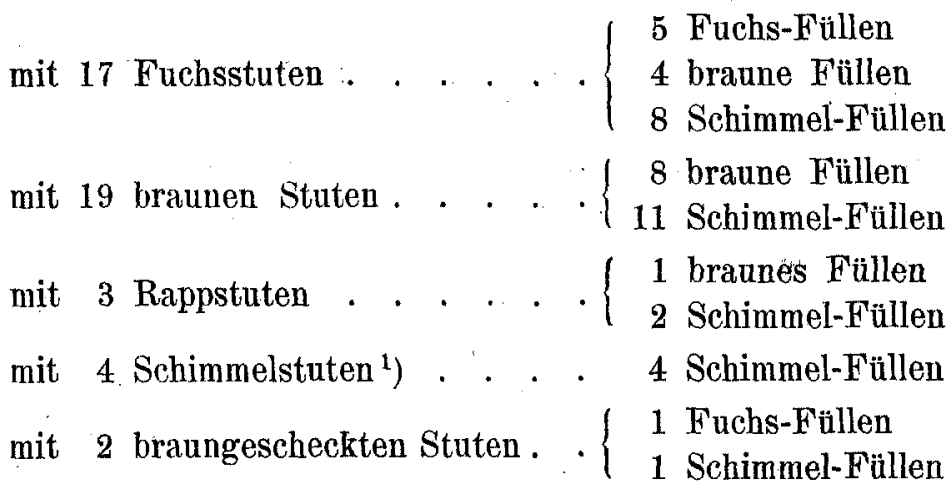

Da der Hengst bei drei Paarungen mit Fuchsstuten sowohl Fuchsfüllen wie braune Füllen gegeben hat, muß er $\mathbf{S}$ heterozygotisch enthalten haben. $\mathrm{Da} B$ er mit einer schwarzen Stute ein braunes Füllen gezeugt hat, beweist, daß er $\mathbf{R}$ mindestens einmal enthält, was im übrigen auch schon von vornherein gegeben ist, da Hads Herreds Munkedal ein Sohn von 839 Aldrup-Munkedal ist, der, wie früher gezeigt, die Formel RR ss hatte. Daß der Hengst in keinem Falle schwarze Nachkommen gegeben hat, deutet darauf hin, daß er $\mathbf{R}$ homozygotisch enthält, aber das Material ist nicht groß genug, um zu sağen, daß der Beweis dafür erbracht ist. Wenn $\mathbf{A}$ den Schimmelfaktor bezeichuet, war also $1049 \mathrm{Hads}$ Herreds Munkedals Formel RR Ss A a oder weniger wahrscheinlich RrSs Aa.

Zur Aufklärung der Vererbungsverhältnisse der Eigenschaft "gescheckt" füge ich den nachstehenden Stammbaum bei, der zeigt, daß der Faktor für diese Eigenschaft über die gewöhnlichen Hanptfarben

1) Im Grunde nur zwei Stuten, von denen die eine drei Füllen von Hads Herreds Munkedal hat, die andere eins. 
dominiert, ganz ebenso wie der Schimmelfaktor ${ }^{1}$ ). Die Stammtafel ist rückwärts ausgearbeitet, weshalb sie nicht dentlich genug zeigt, daß gescheckte Tiere, welche ja gewöhnlich Heterozygoten sind, bei der Paarung mit Nichtschecken nur 50\% gescheckte Nachkommenschaft ergeben.

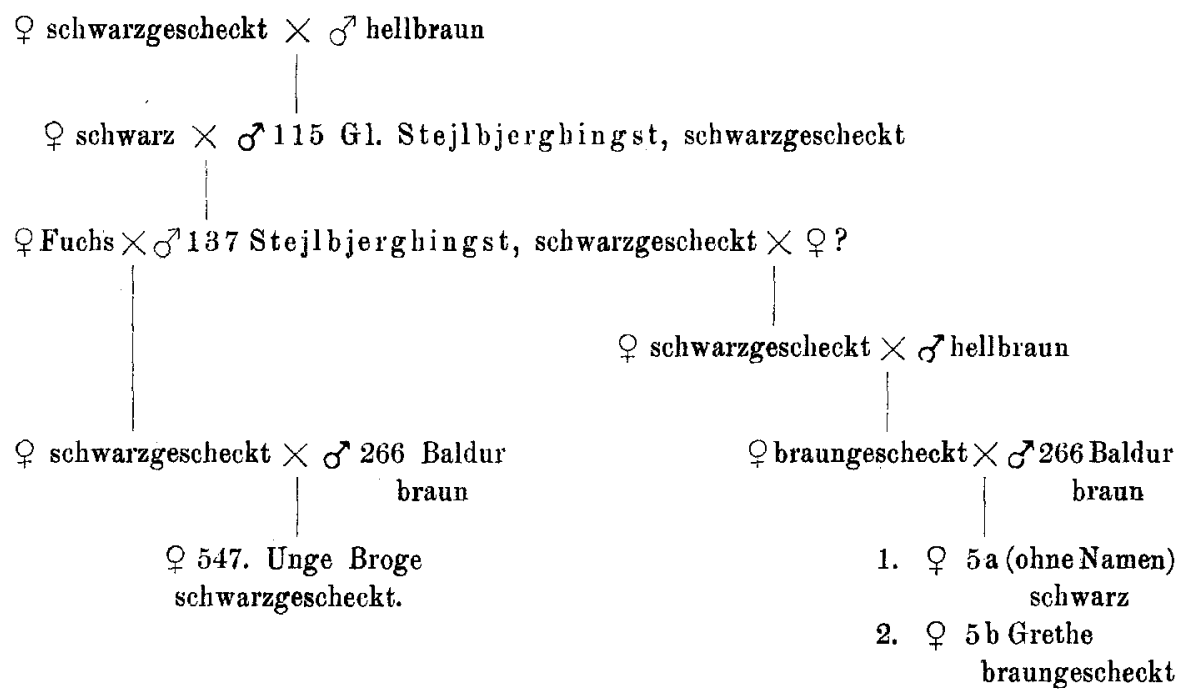

\section{Besprechung der Ergebnisse.}

Das Vorstehende wird gezeigt haben, daß wir in der dänischen, jütländischen Pferderasse - und allem nach gilt dies für die Pferderassen im allgemeinen - bei der Vererbung der Haarfarbe mit einfachen und unkomplizierten Verhältnissen $\mathrm{zu}$ tun haben, und wir wollen im folgenden besprechen, in wiefern diese Erkenntnis am besten ausgenützt werden kann, sowie was geschehen muß, um auch die Verhältnisse für die schon genannten Verdunkelungsfaktoren oder Intensitätsfaktoren aufzuklären, die offenbar auch eine gewisse Rolle spielen.

Der praktische Vorteil der Kenntnis der Farbenformeln eines jeden Stammbuchtieres ist wohl kaum sehr groß, außer für solche Rassen, bei denen gerade besonderer Wert auf die Farbe der Pferde gelegt wird, also nur für die edlen Luxusrassen. Daß aber überall in der Pferdezucht wenigstens etwas Wert auf die Farbe gelegt wird, geht auf der andern Seite deutlich daraus hervor, daß einige Stammbuchtiere die Be-

1) Auch hier scheint es, daß für die Fuchsschecken andere Regeln gelten, welcho jedoch mit Hilfe des vorgefundenen Materials nicht festgelegt werdep können. 
merkung "unerwünschte Farbe" bekommen haben, ebenso wie die häufigen Übersichten über die Farbe der Pferde in den jütländischen Stammbüchern zeigen, daß die Pferdefarbe immerhin etwas ist, wofür man sich interessiert und was in Betracht gezogen wird, sowohl bei der Beurteilung der Qualifikation eines Pferdes im allgemeinen, wie seines Zuchtwerts im besonderen.

Wie aus dem früher Gesagten hervorgeht, wird es eine leichte Sache sein, innerhalb der jütländischen Rasse sowohl fuchsfarbige wie schwarze und braune konstante Unterrassen zu bilden. Allein "mit Hilfe" der zwei in dieser Arbeit besprochenen Faktoren $\mathbf{R}$ und $\mathbf{S}$ können vier konstante Typen hergestellt werden, nämlich ein brauner (RR SS) ein schwarzer (rr SS) und zwei fuchsfarbige (RRss, rr ss). Falls die braune Farbe nur wenig geschätzt ist, kann man sich die Pferde ausschließlich als Rappen und Füchse züchten, auch ohne daß die braune Farbe bei Kreuzungen dieser beiden Farbenschläge anfzutreten brauchte, nämlich, wenn man einfach nur Füchse von der Formel rrss anwendet.

Ich will es unterlassen, Schlüsse zu ziehen oder auch nur schon gezogene Schlüsse eingehend zu kritisieren auf den Zusammenhang zwischen Farbe und den übrigen Eigenschaften bei den jütländischen Pferden. Theoretisch kann das richtig sein, so meint der Stammbuchführer Herr Staatskonsulent Jensen. folgern zu können, daß die besten Zuchttiere unter den Hengsten besonders häufig unter den Füchsen zu finden sind. Eine Koppelung zwischen dem S-Faktor und dem einen oder anderen unerwünschten Faktor würde in diesem Fall wohl die Erklärung geben. Man hat Beweise, daß z. B. Schecken weniger widerstandsfähig gegen gewisse Krankheiten sind als andere, aber daß ein entsprechender nennenswerter Unterschied zwischen Füchsen, Braunen und Rappen besteht, ist mir unbekannt, Staatskonsulent Jensen sagt hierüber im Vorwort" zum Hengststammbuch XIX: „Selbst wenn es unerklärlich ist, scheint doch ein gewisser Zusammenhang zwischen Farbe und Güte (auch als Zuchttier) zu bestehen. So habe ich in der Geschichte des Gestüts Frederiksborg mitgeteilt, als eine Beobachtung aus der Zucht, die dort nach der Farbe betrieben wird, daß die Hengste, deren Farbe von derjenigen der beiden Eltern verschieden war, sich oft in der Zucht $^{1}$ ) auszeichneten. Die gleiche Beobachtung machte ich seiner Zeit

1) Selbst wenn Füchse wirklich besser sind als andere, kann ich keine Berechtigungen zu dieser weitgehenden SchluBfolgerung über den Vorteil von Pferden mit einer von beiden Eltern abweichenden Farbe finden, denn, wie das Schema am Schlub dieser Arbeit zeigt, werden gewib mehr Füchse gezüchtet aus Eltern anderer Farben als 
in der jütländischen Zucht, bevor man richtig auf die Typenzucht gekommen war. . . . . ich bin ständig geneigt, es als ein Plus anzurechnen, weun ein guter Fuchshengst aus Eltern mit anderer Farbe fällt." "Wenn" man nun weiß, daB mindestens zehnmal soviel Stuten als Hengste aufgenommen werden, so daß diese durchschnittlich von besserer Qualität als die Stuten sind, und daß die meisten Hengste Füchse sind, sowie daß das männliche Geschlecht zweifellos das am höchsten entwickelte der beiden Geschlechter ${ }^{1}$ ) ist. so scheint es berechtigt zu schließen, daß die Fuchsfarbe die vorteilhafteste innerhalb der jütländischen Rasse ist."

Es mag ja sein, daß der Wunsch nach einer bestimmten Farbe bei den Pferden nur eine harmlose Modesache ist, aber es ist immerhin eine Sache, die jeden Pferdezüchter angeht, ebenso wie es im allgemeinen von einer gewissen Bedeutung ist, ob der Pferdebestand einer Gegend von unschöner oder aber von hübscher einheitlicher Färbung ist. Die Ästhetik hat wohl auch hier ihre Berechtigung. Daß die Sache mindestens teilweise sich so verhält, daß also die Mode eine Rolle spielt, daranf deutet der folgende Auspruch von Staatskonsulent Jensen (Stutenstammbuch XVII, 1918). Der Verfasser sagt, es gebe eine "Zunahme der Fuchsfarbe auf Kosten der übrigen, deren Ursache darin gesucht werden müsse, daß Fuchs gepaart mit Fuchs immer wieder Fuchs gibt, während Fuchsfüllen auch aus braunen, schwarzen und Schimmeleltern fallen können". "Diese Zunahme der roten Farbe befriedigt ja die herrschende Mode, die verlangt, daß die jütländischen Pferde Füchse sein sollen, und ist deshalb gern gesehen."

Während Staatskonsulent Jensen sicher in seinem Recht ist, wenn er von dem Wunsch der Mode spricht, so ist es unrichtig, die Zunahme des Prozentsatzes der roten Tiere so zu erklären, wie er es tut, auch wenn die Erklärung ansprechend klingen mag. Die Konsequenz wäre dann ja, daß rezessive Typen stets in allen Arten von, Kulturen an Zahl zunehmen! Die Erklärung liegt vielmehr darin, daß eine

Braune und Rappen; aber die Rappen und Braunen, welche aus Eltern anderer Farbe gefallen sind, ergeben zusammen eine noch gröBere Zahl als Füchse. Der SchluB kann deshalb kaum richtig sein. - $\mathrm{Ob}$ ein $\mathbf{r} \mathbf{S} \mathrm{Ss}$ Individuum z. B. von zwei braunen Eltern stammt, oder von zwei schwarzen oder von einem braunen und einem schwarzen oder einem fuchsfarbenen und einem schwarzen oder einem fuchsfarbenen und einem braunen, kann im übrigen die Qualität eines Tieres nicht berühren.

ö. W.

1) Da die Vererbung der Farbenfaktoren nicht geschlechtsbegrenzt ist, finde ich kein Argument in diesen letaten Satz.

o. W. 
ungleiche Selektion von Tieren der verschiedenen Farben vorgenommen wird - entweder deswegen, weil Füchse faktisch im Durchschnitt besser sind als die anderen - oder weil man einfach aus rein ästhetischen Gründen Füchse bevorzugt. Falls keine solche Selektion stattfände, würde das Zahlenverhältnis zwischen Füchsen, Braunen und Rappen stets gleich bleiben.

In der Hauptsache ist es, wenigstens periodenweise, nur eine ganz kleine Anzahl von Hengsten, sogenannte "Matadore", welche die Stammbücher mit Material versehen. Es kann z. B. in einem gegebenen Jahr ein einziger Hengst der Vater von 13\% aller in den Stammbüchern aufgeführten Tiere sein, und nur eine kleine Zahl von Hengsten erzeugte zusammen allein schon 50\% aller aufgeführten Tiere. Unter diesen Umständen muß es selbstverständlich ausschlaggebend für die Stammbücher der folgenden Jahre sein, welche Farbe diese Matadore haben. Es ist bekannt, daß die "Munkedaler", die in der Hauptsache Füchse waren, eine ganz gewaltige Rolle in der jütländischen Pferdezucht gespielt haben. Es ist deshalb kein Wunder, daß der Prozentsatz von Füchsen steigt in dem Maß, wie die Nachkommen der Munkedaler sich in den Stammbüchern ausbreiten.

Die bisherige Kenntnis der Praktiker von der Vererbung der Pferdefarbe ist sehr gering gewesen. In den dänischen Stammbüchern hat man sich im wesentlichen an die statistische Übersicht gehalten und damit in der Hauptsache die alte Erfahrung bestätigt, daß zwei Füchse stets Fuchsfohlen geben, und daß schwarze Eltern nur schwarze oder fuchsfarbene geben, aber keine braunen. Die theoretische Seite hat man vernachlässigt. Die Beiträge der Theoretiker auf diesem Gebiet sind freilich auch nicht alle sehr wertvoll gewesen, und es ist mir bekannt, daß James Wilson's "The Principles of Stock-Breeding*, 1912, welches in das Dänische von Prof. Harald Goldschmidt übersetzt ist, mit seinen falschen Angaben über Vererbung der Pferdefarben dazu beigetragen hat, das Zutrauen der Pferdezüchter zur Wissenschaft zu schwächen.

Einige andere Beobachtungen, die über die eben gemachten hinaus gehen, sind aber doch von Staatskonsulent Jensen gemacht worden. So hat er richtig erkannt, daß die braune Farbe eine Mischung von fuchsfarbig und schwarz ist, und ébenso, daß der Fuchshengst 839 Aldrup Munkedal nië schwarze Nachkommen gegeben hat. Fr schreibt unter anderem im Stutenstammbuch XIII, 1914, "die Regeln für die Vererbung der Pferdefarben variieren sehr, so kennt man Rückschläge zu Schimmel kaum, wohl aber zu gelb. Im ganzen genommen scheint ein Rückschlag 
über das zweite Glied hinaus stark abzunehmen. Auch z.Z. weiß man sehr wenig über diese wichtige Sache, und es ist zweifelhaft, ob man irgend eine Erklärung hierfür finden wird, wenn nicht das Mendelsche Spaltungsgesetz den Weg zeigen kann."

Daß das Mendelsche Gesetz den Weg zeigen kann, das nachzuweisen war der Zweck der vorliegenden Arbeit, wie auch der früheren Arbeiten von anderen Autoren, und $\mathrm{da} \beta$ es darin noch weiter wird führen können, daran hege ich keinen Zweifel, aber es ist zu diesem Zweck notwendig, daß die Stammbücher in viel höherem Grad als bisher bei der Beschreibung der Pferdefarben ins einzelne gehen, am besten mit Hilfe einer Farbentafel.

Wenn Staatskonsulent Jensen im Statenstammbuch XIV, 1915 schreibt, „die Farbe bei den aufgenommenen Stuten ist anders geworden, so daß es jetzt $65 \%$ Füchse sind, was einen Zuwachs von $3 \%$ bedeutet. Leider scheinen sie aber nicht schöner zu werden, indem die helle, gelbliche Farbe mit heller Mähne und Schwanz sehr verbreitet ist, ebenso wie ein dunkel-schmutzigroter Ton" - so ist man berechtigt zu schließen, $\mathrm{da} ß$ heute einige wenig gewünschte Fuchs-Biotypen dabei sind, unter den Pferden die Oberhand zu bekommen. - Noch ist wie gesagt, der Vererbungsforscher anßerstand, den Praktikern zu helfen, soweit es sich um Abstufungen innerhalb der verschiedenen Hauptfarben handelt, aber es wird zweifellos auch gelingen, mit diesen Schattierungsfaktoren ins reine zu kommen, wenn nur die Stammbuchführer das ihrige dazu beitragen, daßein Material zusammenkommt, das zurBeurteilung der Zahl und Wirkungsweise dieser Faktoren ausreicht. Das gleiche gilt für die Abzeichen. Nach den Ergebnissen der parallelen Untersuchungen mit anderen Tieren zu schließen, vor allem mit Ratten, sind die Größenverhältnisse der Abzeichen phänotypisch sehr variabel und genotypisch abhängig von mehreren Faktoren. Eine spezielle Untersuchung über ihre Vererbungsverhältnisse würde jedoch sicher diese Verhältnisse für die Pferde klar legen können.

Es würde in vielen Fällen für die Stammbuchführer eine einfache Sache sein, selbst den Stammbuchtieren die Farbenformel beizufügen, so wie ich es in dieser Abhandlung getan habe. Für junge Tiere, deren Nachkommen man nicht kennt, wird das ganz gewiß nur selten möglich sein. Die Arbeit müßte mit der Untersuchung von älteren Tieren begonnen werden, die eine zahlreiche Nachkommenschaft haben, und wenn erst deren Formeln berechnet sind, können diejenigen der späteren Tiere dann umso leichter gefunden werden. 
Eine große Hilfe würde es sein, wenn die Farben beider Eltern in die Beschreibung jedes Stammbuchtieres aufgenommen werden könnten. Erst seit dem Jahre 1916 ist im Stutenstammbuch XV die Farbe der Mutter mit aufgenommen, „um die Ausarbeitung einer Statistik über die Vererbung der Farben zu erleichtern". In den Hengststammbüchern ist die Farbe der Mutter stets angegeben worden. Für den Vater wird aber leider niemals etwas anderes als Name und Nummer angegeben.

Es müßte eine dankbare Aufgabe, wenn auch nicht gerade eine der wichtigsten, für die Stammbuchführer und Pferdezüchter sein, so genau als möglich die Formel eines jeden Tieres angeben zu können, so daß man mit größter Sicherheit gerade diejenigen Paarungen vornehmen könnte, welche Nachkommen von der gewünschten Farbe ergeben.

Zum Schluß füge ich die Statistik bei, welche ich über die Farbe der Nachkommen von Paarungen von Tieren der drei Hauptfarben ausgearbeitet habe. Die reziproken Paarungen von Tieren verschiedener Farbe sind dabei getrennt gehalten.

Tabelle über die Farbe der Nachkommen nach verschiedenen Paarungen.

\begin{tabular}{c|c|c|c}
\hline \hline Nachkommen & o Fuchs $\times \sigma^{7}$ Fuchs & o Braun $\times \sigma^{7}$ Braun & o Rappe $\times \sigma^{\top}$ Rappe \\
\hline Füchse & 2473 & 396 & 21 \\
Braune & $\left.7^{1}\right)$ & 1480 & 0 \\
Rappen & 0 & 132 & 133
\end{tabular}

\begin{tabular}{c|c|c|c}
\hline \hline Nachkommen & of Fuchs $\times$ o Braun & o Fuchs $\times$ o Rappe & o Braun $\times$ o Rappe \\
\hline Füchse & 642 & 114 & 79 \\
Braune & 519 & 97 & 372 \\
Rappen & 56 & 50 & 138
\end{tabular}

\begin{tabular}{l|c|c|c}
\hline Nachkommen & Q Braun $\times \sigma^{7}$ Fuchs & Q Rappe $\times \sigma^{7}$ Fuchs & R Rappe $\times \sigma^{7}$ Braun \\
\hline Fïchse & 1133 & 270 & 61 \\
Braune & 1250 & 193 & 207 \\
Rappen & 121 & 120 & 106
\end{tabular}

1) Wegen dieser 7 Individuen vgl. Seite 10. 


\section{Zusammenfassung.}

In der vorliegenden Arbeit werden die Vererbungsverhältnisse der Pferdefarben an der Hand des Stammbuchmaterials über die Pferde der dänischen jütländischen Pferderasse untersucht. Die Untersuchung umfaßt über 10000 Stammbuchtiere.

Die Untersuchung bezieht sich in der Hauptsache auf Pferde der drei Hauptfarben: Fuchs, Branner, Rappe, außerdem Schimmel und Schecken.

Walther und Wentworth haben Theorien aufgestellt, welche z. T. richtig sind, aber ihre Bezeichnungsweise für die Wirkung der Faktoren macht die Theorien unnötig verschnörkelt.

Für die drei Hauptfarben rechnen die beiden genannten Autoren mit drei Faktoren (oder Faktorenparen), während man nur zwei anzunehmen braucht, nämlich $\mathbf{R}$ Faktor für Fuchsfarbe, $\mathbf{S}$ Faktor für schwarze Farbe. Tiere, welche beide Faktoren haben, sind braun. Bei Tieren, welche weder $\mathbf{S}$ noch $\mathbf{R}$ enthalten, kommt die ganze rezessive Pferdefarbe zum Vorschein, welche ebenfalls fuchsfarbig ist.

Mit Hilfe der Stammbücher werden Hengste mit den verschiedenen Formeln nachgewiesen.

In Übereinstimmung damit, daß schwarze Pferde in der jütländischen Rasse selten sind, wird gezeigt, daß die Formeln der braunen Pferde gewöhnlich RrSs oder RRSs, selten dagegen RrSS und RRSS sind. Es wird ferner gezeigt, daB zu einem gegebenen Zeitpunkt ungefähr $40 \%$ der brannen Pferde homozygotisch in $\mathbf{R}$, aber nur zirka $10 \%$ homozygotisch in $\mathbf{S}$ waren.

Einer gegebenen Formel entspricht zwar eine bestimmte Hauptfarbe, aber nicht eine bestimmte Nuance. Es gibt besondere Nuancierungsfaktoren, deren Zahl und Wirkungsweise noch nicht klar gelegt ist ebensowenig wie die der Faktoren, die für die Größe und Verteilung der Abzeichen bestimmend sind.

In Übereinstimmung mit anderweitigen früheren Untersuchungen wird je ein dominierender Faktor für Schimmelung und für Scheckung nachgewiesen. Die stets zunehmende Häufigkeit der Füchse in der jütländischen Rasse wird besprochen und es wird ferner angegeben, was weiter geschehen muß, um die noch unklaren Punkte klarzulegen.

Carlsberg Laboratorinm, Kopenhagen, den 21. November 1919. 


\section{Literatur.}

Wegen älterer Literatur vergleiche A. Lang: Die experimentelle Vererbungslehre in der Zoologie seit 1900, 1. Hälfte, Jena 1914.

Sturtevant, A. H., A Critical Examination of Recent Studies on Color Inheritance in Horses. Journ. of Gen., Bd. II, 1912.

Walther, Ad. R, Beiträge zur Kenntnis der Vererbung der Pferdefarben. Hannover 1912.

Wentworth, E. N., Color Inheritance in the Horse. Zeitschr. f. ind. Abst.- und Vererbgsl., Bd. XI, 1914.

Wilson, James, Inheritance of the Dun Coat Colour in Horses. Scientif. Proc. of the Royal Dublin Soc., Bd. XII (N. S.), 1910. 